\title{
The deuterium fractionation of water on solar-system scales in deeply-embedded low-mass protostars ${ }^{\star}$
}

\author{
M. V. Persson ${ }^{1,2,3}$, J. K. Jørgensen ${ }^{2,1}$, E. F. van Dishoeck ${ }^{3,4}$, and D. Harsono ${ }^{3}$ \\ ${ }^{1}$ Centre for Star and Planet Formation, Natural History Museum of Denmark, University of Copenhagen, Øster Voldgade 5-7, \\ 1350 Copenhagen K, Denmark \\ e-mail: magnusp@strw. leidenuniv.nl \\ 2 Niels Bohr Institute, University of Copenhagen, Juliane Maries Vej 30, 2100 Copenhagen Ø, Denmark \\ ${ }^{3}$ Leiden Observatory, Leiden University, PO Box 9513, 2300 RA Leiden, The Netherlands \\ ${ }^{4}$ Max-Planck Institute für extraterrestrische Physik (MPE), Giessenbachstrasse, 85748 Garching, Germany
}

Received 14 October 2013 / Accepted 3 February 2014

\section{ABSTRACT}

\begin{abstract}
Context. The chemical evolution of water through the star formation process directly affects the initial conditions of planet formation. The water deuterium fractionation $\left(\mathrm{HDO} / \mathrm{H}_{2} \mathrm{O}\right.$ abundance ratio) has traditionally been used to infer the amount of water brought to Earth by comets. Measuring this ratio in deeply-embedded low-mass protostars makes it possible to probe the critical stage when water is transported from clouds to disks in which icy bodies are formed.

Aims. We aim to determine the $\mathrm{HDO} / \mathrm{H}_{2} \mathrm{O}$ abundance ratio in the warm gas in the inner $150 \mathrm{AU}$ for three deeply-embedded low-mass protostars NGC 1333-IRAS 2A, IRAS 4A-NW, and IRAS 4B through high-resolution interferometric observations of isotopologues of water.

Methods. We present sub-arcsecond resolution observations of the $3_{1,2}-2_{2,1}$ transition of HDO at $225.89672 \mathrm{GHz}$ in combination with previous observations of the $3_{1,3}-2_{2,0}$ transition of $\mathrm{H}_{2}^{18} \mathrm{O}$ at $203.40752 \mathrm{GHz}$ from the Plateau de Bure Interferometer toward three low-mass protostars. The observations have similar angular resolution $\left(0.7-1{ }^{\prime \prime} .3\right)$, probing scales $R \lesssim 150$ AU. In addition, observations of the $2_{1,1}-2_{1,2}$ transition of HDO at $241.561 \mathrm{GHz}$ toward IRAS $2 \mathrm{~A}$ are presented to constrain the excitation temperature. A direct and model independent $\mathrm{HDO} / \mathrm{H}_{2} \mathrm{O}$ abundance ratio is determined for each source and compared with $\mathrm{HDO} / \mathrm{H}_{2} \mathrm{O}$ ratios derived from spherically symmetric full radiative transfer models for two sources.

Results. From the two HDO lines observed toward IRAS 2A, the excitation temperature is found to be $T_{\text {ex }}=124 \pm 60 \mathrm{~K}$. Assuming a similar excitation temperature for $\mathrm{H}_{2}^{18} \mathrm{O}$ and all sources, the $\mathrm{HDO} / \mathrm{H}_{2} \mathrm{O}$ ratio is $7.4 \pm 2.1 \times 10^{-4}$ for IRAS $2 \mathrm{~A}, 19.1 \pm 5.4 \times 10^{-4}$ for IRAS 4A-NW, and $5.9 \pm 1.7 \times 10^{-4}$ for IRAS $4 \mathrm{~B}$. The abundance ratios show only a weak dependence on the adopted excitation temperature. The abundances derived from the radiative transfer models agree with the direct determination of the $\mathrm{HDO} / \mathrm{H}_{2} \mathrm{O}$ abundance ratio for IRAS 16293-2422 within a factor of 2-3, and for IRAS 2A within a factor of 4; the difference is mainly due to optical depth effects in the HDO line.

Conclusions. Our $\mathrm{HDO} / \mathrm{H}_{2} \mathrm{O}$ ratios for the inner regions (where $T>100 \mathrm{~K}$ ) of four young protostars are only a factor of 2 higher than those found for pristine, solar system comets. These small differences suggest that little processing of water occurs between the deeply embedded stage and the formation of planetesimals and comets.
\end{abstract}

Key words. astrochemistry - stars: formation - ISM: abundances - protoplanetary disks - stars: general

\section{Introduction}

Water is a very important molecule through all stages of star and planet formation. In dense clouds, it is one of the dominant reservoirs of oxygen, both as a gas at high temperatures or as ice in grain mantles in cold regions. It also aids in the sticking of grains outside the snow line, and is crucial for the emergence of life on Earth. This makes it vital to understand the chemical evolution of water during the different stages of star formation, and to identify the mechanism by which it was brought to the Earth and Earth-like planets.

One way to gain insight into the chemical evolution of water is to compare the level of deuterium fractionation in water (the $\mathrm{HDO} / \mathrm{H}_{2} \mathrm{O}$ ratio) at various stages of star and planet formation. In our own solar system, several measurements of the $\mathrm{HDO} / \mathrm{H}_{2} \mathrm{O}$ ratio ${ }^{1}$ exist for long-period comets from the Oort cloud (Mumma \& Charnley 2011, and references therein).

* Based on observations carried out with the IRAM Plateau de Bure Interferometer.

$1 \mathrm{D} / \mathrm{H}=0.5 \times \mathrm{HDO} / \mathrm{H}_{2} \mathrm{O}$.
Up until recently the mean $\mathrm{HDO} / \mathrm{H}_{2} \mathrm{O}$ ratio for these objects was $6.4 \pm 1.0 \times 10^{-4}$ (Villanueva et al. 2009; Jehin et al. 2009), significantly above the value for the Earth's oceans $3.114 \pm$ $0.002 \times 10^{-4}$ (VSMOW $^{2}$, de Laeter et al. 2003, and references therein). Both values are well above the protosolar nebula value of $2 \times \mathrm{D} / \mathrm{H}=0.42 \pm 0.08 \times 10^{-4}$ (Geiss \& Gloeckler 1998; Lellouch et al. 2001) indicating that deuterium fractionation of water has taken place. Because of the observed difference between the Earth and comets, it has been argued that only a small fraction $(\leq 10 \%)$ of the Earth's water could have its origin in comets (Morbidelli et al. 2000; Drake 2005).

The Jupiter family comet Hartley 2 observed with the Herschel Space Observatory was found to have a $\mathrm{HDO} / \mathrm{H}_{2} \mathrm{O}$ ratio of $3.2 \pm 0.5 \times 10^{-4}$, significantly lower than the value for Oort cloud comets, and comparable to VSMOW (Hartogh et al. 2011). Jupiter family comets have relatively short periods ( $\$ 20$ years), with orbits more or less confined to the ecliptic plane, and originate in the Kuiper belt (Duncan et al. 2004; Mumma \& Charnley 2011). Oort cloud comets have longer

2 Vienna Standard Mean Ocean Water. 
periods, orbits distributed isotropically, and originate in the Oort cloud. In 2012 Bockelée-Morvan et al. observed the Oort cloud comet Garradd with Herschel and deduced a $\mathrm{HDO} / \mathrm{H}_{2} \mathrm{O}$ ratio of $4.12 \pm 0.44 \times 10^{-4}$. This is significantly lower than previous measurements of Oort cloud comets. Recently, Lis et al. (2013) measured the $\mathrm{HDO} / \mathrm{H}_{2} \mathrm{O}$ ratio in the Jupiter family comet Honda-Mrkos-Pajdusakov (HMP) using Herschel to $<4.0 \times 10^{-4}$. These latest measurements indicate a certain range in the water deuterium fractionation in comets, possibly dependent on the precise formation location and subsequent migration, in which Jupiter family comets no longer have a distinctly lower $\mathrm{HDO} / \mathrm{H}_{2} \mathrm{O}$ ratio than Oort cloud comets. Simulations suggest that the deuterium fractionation in the protosolar nebula disk increases with distance from the forming sun (Hersant et al. 2001; Kavelaars et al. 2011) - but whether the observed ratios are consistent with these simulations is still unclear.

Measurements of the water deuterium fractionation toward protostars provide a different perspective. By determining the $\mathrm{HDO} / \mathrm{H}_{2} \mathrm{O}$ ratio of material that enters protoplanetary disks, it is possible to set the initial level of deuterium fractionation in comet forming zones as input for any subsequent evolutionary models. So far, determinations of $\mathrm{HDO} / \mathrm{H}_{2} \mathrm{O}$ have given a wide range of results. Parise et al. (2003) used ground-based infrared observations of the stretching bands of $\mathrm{OH}$ and OD in water ice in the cold outer parts of protostellar envelopes and found $u p$ per limits ranging from $0.5 \%$ to $2 \%$ for the $\mathrm{HDO} / \mathrm{H}_{2} \mathrm{O}$ ratios. Studies using gaseous HDO lines differ in the interpretation, with cold $\mathrm{HDO} / \mathrm{H}_{2} \mathrm{O}$ ratios derived from single-dish data ranging from cometary values $\left(2 \times 10^{-4}\right.$, Stark et al. 2004), to a few $\times 10^{-2}$ (Parise et al. 2005; Liu et al. 2011; Coutens et al. 2012). In the inner warm $(T>100 \mathrm{~K})$ region, $\mathrm{HDO} / \mathrm{H}_{2} \mathrm{O}$ ratios $\geq 1 \%$ have also been inferred based on analysis of many HDO and water lines ( $E_{\mathrm{u}}$ up to $450 \mathrm{~K}$ ) (Liu et al. 2011; Coutens et al. 2012). In contrast, Jørgensen \& van Dishoeck (2010b) derived a HDO/ $\mathrm{H}_{2} \mathrm{O}$ ratio toward the Class 0 protostar NGC 1333-IRAS 4B of $<6.4 \times 10^{-4}$ from the interferometric Submillimeter Array (SMA) and Plateau de Bure (PdBI) observations, up to two orders of magnitude lower.

There are two main issues with determining the water abundances in the warm gas in the inner few hundred au. First, the relatively large beam sizes $\left(>10^{\prime \prime}\right)$ of single-dish telescopes are more sensitive to extended emission than the small $\sim 1^{\prime \prime}$ scales on which the warm water is found. Second, many observed water lines suffer from high optical depths, even for isotopologues, making it difficult to infer reliable column densities and abundances for $\mathrm{H}_{2} \mathrm{O}$ in the warm $(>100 \mathrm{~K})$ gas. Indeed, Visser et al. (2013) find that the $3_{1,2}-3_{0,3}$ lines $\left(E_{\mathrm{u}}=249 \mathrm{~K}\right)$ of $\mathrm{H}_{2}^{16} \mathrm{O}, \mathrm{H}_{2}^{18} \mathrm{O}$ and even $\mathrm{H}_{2}^{17} \mathrm{O}$ observed by the HIFI instrument (de Graauw et al. 2010) aboard Herschel probably show optically thick emission on $R \sim 100$ AU scales in the well-studied Class 0 protostar NGC1333-IRAS 2A. Taking the optical depth properly into account lowers the $\mathrm{HDO} / \mathrm{H}_{2} \mathrm{O}$ ratio for IRAS $2 \mathrm{~A}$ from $\geq 0.01$ to $1 \times 10^{-3}$ (Visser et al. 2013).

Recent high-resolution interferometric ground-based observations have made it possible to estimate the abundances of both $\mathrm{H}_{2} \mathrm{O}$ and $\mathrm{HDO}$ in the warm gas on small scales. Jørgensen \& van Dishoeck (2010a) targeted the $\mathrm{H}_{2}^{18} \mathrm{O} 3_{1,3}-2_{2,0}$ line at $203 \mathrm{GHz}$ with PdBI, which has a much lower Einstein $A$ coefficient than the lines observed with Herschel and thus suffers less from optical depth effects. Using SMA data of the HDO $3_{1,2}-2_{2,1}$ transition at $225 \mathrm{GHz}$, the above mentioned $\mathrm{HDO} / \mathrm{H}_{2} \mathrm{O}$ limit of $<6.4 \times$ $10^{-4}$ was found toward NGC 1333-IRAS 4B (Jørgensen \& van Dishoeck 2010b). Coutens et al. (2013) obtained values of $4-30 \times 10^{-4}$ for IRAS $4 \mathrm{~A}$ and $1-37 \times 10^{-4}$ for IRAS 4B using new Herschel data combined with the $\mathrm{H}_{2}^{18} \mathrm{O}$ column densities found by Persson et al. (2012) based on interferometric data.

Taquet et al. (2013) derived the warm $\mathrm{HDO} / \mathrm{H}_{2} \mathrm{O}$ ratios for IRAS 2A and IRAS 4A using the determinations of the $\mathrm{H}_{2}^{18} \mathrm{O}$ column density from Persson et al. (2012) and lower spatial- and spectral-resolution interferometric observations targeting the HDO $4_{2,2}-4_{2,3}$ transition $\left(143.7 \mathrm{GHz}, E_{\mathrm{u}}=316.2 \mathrm{~K}\right.$ ) together with single dish (Liu et al. 2011) observations of HDO. Using non-local thermal equilibrium (non-LTE) large velocity gradient (LVG) analysis, ratios in the range of $30-800 \times 10^{-4}$ for IRAS $2 \mathrm{~A}$ and $50-300 \times 10^{-4}$ for IRAS 4A were obtained, depending on the assumed density, with the high-density $\left(n_{\mathrm{H}_{2}}=\right.$ $1 \times 10^{8} \mathrm{~cm}^{-3}$ ) solution giving the lowest ratios. Finally, using Atacama Large Millimeter/submillimeter Array (ALMA) and SMA observations, Persson et al. (2013) estimated the $\mathrm{HDO} / \mathrm{H}_{2} \mathrm{O}$ ratio of the warm water toward IRAS 16293-2422 A to $9.2 \pm 2.6 \times 10^{-4}$.

Most of these new values, summarized in Table A.1, are significantly lower than the previous estimates of a few percentage points, indicating that the deuterium fractionation in warm water may not be as enhanced as previously thought.

In order to settle the question of the $\mathrm{HDO} / \mathrm{H}_{2} \mathrm{O}$ abundance ratios in warm gas, we have obtained high spatial resolution interferometric observations of HDO to complement previous high quality data of $\mathrm{H}_{2}^{18} \mathrm{O}$ for three sources. The observations are sensitive to scales comparable to disks $\left(\sim 11^{\prime \prime} 3, R \lesssim 150\right.$ AU), allowing us to determine the $\mathrm{HDO} / \mathrm{H}_{2} \mathrm{O}$ ratio in the innermost parts of young protostars where the disk is forming.

This paper presents observations of HDO $3_{1,2}-2_{2,1}$ at $225.9 \mathrm{GHz}$ toward the three well-known deeply-embedded low-mass protostars in the NGC 1333 region of the Perseus molecular cloud (Jennings et al. 1987; Sandell et al. 1991 IRAS 2A, IRAS 4A, and IRAS 4B in the following) at a distance of $250 \mathrm{pc}$ (Enoch et al. 2006). The three sources are ideal to compare because of their similar masses, luminosities, and location (Jørgensen et al. 2006). Persson et al. (2012) presented observations of the $\mathrm{H}_{2}^{18} \mathrm{O} 3_{1,3}-2_{2,0}$ transition at $203.4 \mathrm{GHz}$ toward the same sources. With these two datasets we can deduce a $\mathrm{HDO} / \mathrm{H}_{2} \mathrm{O}$ ratio in the warm gas in the inner $150 \mathrm{AU}$ radius using the same method and similar sensitivity and resolution as for IRAS 16293-2422, bringing the total number of low-mass protostars for which high quality data exist to four. In addition, observations of HDO $2_{1,1}-2_{1,2}$ at $241.6 \mathrm{GHz}$ toward IRAS $2 \mathrm{~A}$ were acquired to empirically constrain the excitation temperature. The paper is laid out as follows. Section 2 describes the details of the various observations. Sections 3 and 4 present the results and analysis - including intensities, column densities, spectra, maps, and line radiative transfer modeling of the observed emission. In Sect. 5 we discuss the deduced $\mathrm{HDO} / \mathrm{H}_{2} \mathrm{O}$ ratio and compare it to other studies of protostars and with solar system objects.

\section{Observations}

Three low-mass protostars, IRAS 2A, IRAS 4A, and IRAS 4B in the embedded cluster NGC 1333 were observed using the PdBI. All sources were observed with two different receiver setups. One setup was tuned to the $\mathrm{H}_{2}^{18} \mathrm{O} 3_{1,3}-2_{2,0}$ transition at $203.40752 \mathrm{GHz}$, for details about those observations see Jørgensen \& van Dishoeck (2010a) and Persson et al. (2012). In the other setup, the receivers were tuned to the HDO $3_{1,2}-2_{2,1}$ transition at $225.89672 \mathrm{GHz}$. IRAS 2A was observed in the $C$ configuration on 27 and 28 November 2011 ( 8 h; including calibration) and the B configuration on 12 March 2012 
Table 1. Parameters for the observations tuned to $\mathrm{HDO}$ at $225.9 \mathrm{GHz}$ and $241.6 \mathrm{GHz}$.

\begin{tabular}{llll}
\hline \hline Source & $\begin{array}{l}\text { Beam }^{a} \\
{\left[{ }^{\prime \prime}\right]}\end{array}$ & $\begin{array}{l}\text { PA } \\
{\left[{ }^{\circ}\right]}\end{array}$ & $\begin{array}{l}\text { RMS } \\
{\left[{ }^{b}\right]}\end{array}$ \\
\hline IRAS 2A & $1.3 \times 1.0$ & 23.3 & 16.0 \\
IRAS 2A $^{c}$ & $1.2 \times 0.9$ & 13.3 & 10.5 \\
IRAS 4A & $1.1 \times 0.8$ & 11.3 & 12.5 \\
IRAS 4B & $1.1 \times 0.8$ & 10.6 & 10.2 \\
\hline
\end{tabular}

Notes. ${ }^{(a)}$ Major $\times$ minor axis. ${ }^{(b)}$ Units of mJy beam ${ }^{-1}$ channel $^{-1}{ }^{(c)}$ For the $241.6 \mathrm{GHz}$ observations.

( $2 \mathrm{~h})$. IRAS $4 \mathrm{~A}$ and IRAS $4 \mathrm{~B}$ were observed in track sharing mode in the B configuration on 12 March $2012(4 \mathrm{~h})$, and $\mathrm{C}$ on 15, 27, and 21 March and on 2 April ( $3 \mathrm{~h})$. For IRAS 2A the combined dataset covers baselines from 15.8 to $452.0 \mathrm{~m}$ (11.9 to $340.5 \mathrm{k} \lambda)$, for IRAS 4A from 15.0 to $452.0 \mathrm{~m}(11.3$ to $340.5 \mathrm{k} \lambda)$, and for IRAS 4B from 16.3 to $451.9 \mathrm{~m}(12.3$ to $340.5 \mathrm{k} \lambda$ ). The correlators were set up with one unit with a bandwidth of $40 \mathrm{MHz}\left(47.6 \mathrm{~km} \mathrm{~s}^{-1}\right)$ centered on the frequency of the HDO line $(225.89672 \mathrm{GHz})$, providing a spectral resolution on 460 channels of $0.087 \mathrm{MHz}\left(0.104 \mathrm{~km} \mathrm{~s}^{-1}\right)$ width $^{3}$.

The data were calibrated and imaged using the CLIC and MAPPING packages, part of the IRAM GILDAS software. Regular observations of the nearby, strong quasars $0333+321$, $3 \mathrm{C} 84$, and $2200+420$ or $1749+096$ were used to calibrate the complex gains and bandpass, while MWC349 and 0333+321 were observed to calibrate the absolute flux scale. During the calibration procedure integrations with significantly deviating amplitude and or phases were flagged and the continuum was subtracted before Fourier transformation of the line data.

In addition to these observations, the HDO $2_{1,1}-2_{1,2}$ line at $241.56155 \mathrm{GHz}$ was observed toward IRAS $2 \mathrm{~A}$ on 7 and 17 November 2012 in the $\mathrm{C}$ configuration for 8 hours covering baselines from 15.5 to $175.8 \mathrm{~m}$ (12.5 to $141.7 \mathrm{k} \lambda)$. The correlators were set up with one unit covering $80 \mathrm{MHz}\left(89.0 \mathrm{~km} \mathrm{~s}^{-1}\right)$ with a spectral resolution of $0.174 \mathrm{MHz}\left(0.1939 \mathrm{~km} \mathrm{~s}^{-1}\right)$ on 460 channels. The same calibration steps as described above were followed.

The resulting beam sizes using natural weighting and other parameters for the observations are given in Table 1 . The field of view is roughly $22^{\prime \prime}$ (HPBW) at $225.9 \mathrm{GHz}$ and the continuum sensitivity is limited by the dynamical range of the interferometer. The uncertainty in fluxes is dominated by the accuracy of the flux calibration, typically about $20 \%$.

\section{Results}

Both the targeted lines and the continuum are detected with high signal to noise in all three sources (Fig. 1). Elliptical Gaussian fits to the $(u, v)$-plane of the continuum are tabulated in Table 2. The continuum emission from the sources agrees with previous observations (e.g., Jørgensen et al. 2007; Jørgensen \& van Dishoeck 2010a; Persson et al. 2012), following a power-law $\left(F_{v} \propto v^{\alpha}\right)$ with exponents expected from thermal dust continuum emission (i.e., $\alpha \sim 2-3$ ). This shows that the flux calibrations for the different observations are accurate to within roughly $20 \%$.

To identify the lines, the Jet Propulsion Laboratory (JPL, Pickett et al. 1998) and the Cologne Database for Molecular Spectroscopy (CDMS, Müller et al. 2001) line-lists were

\footnotetext{
3 Spectra and maps are available through http://vilhelm.nu
}

Table 2. Parameters of the continuum determined from elliptical Gaussian fits in the $(u, v)$-plane.

\begin{tabular}{llll}
\hline \hline Source & $\begin{array}{l}\text { Intensity } \\
{[\mathrm{Jy}]}\end{array}$ & $\begin{array}{l}\text { PA } \\
{\left[{ }^{\circ}\right]}\end{array}$ & $\begin{array}{l}\text { Size } \\
{\left[{ }^{\prime \prime}\right]}\end{array}$ \\
\hline IRAS 2A & 0.4 & 27.5 & $1.1 \times 10$ \\
IRAS 2A $^{a}$ & 0.5 & 23.7 & $1.3 \times 10$ \\
IRAS 4A-NW & 1.7 & - & 2.2 \\
IRAS 4A-SE & 2.1 & - & 1.6 \\
IRAS 4B & 1.1 & 6.7 & $2.2 \times 1.3$ \\
\hline
\end{tabular}

Notes. ${ }^{(a)}$ For the $241.6 \mathrm{GHz}$ observations.

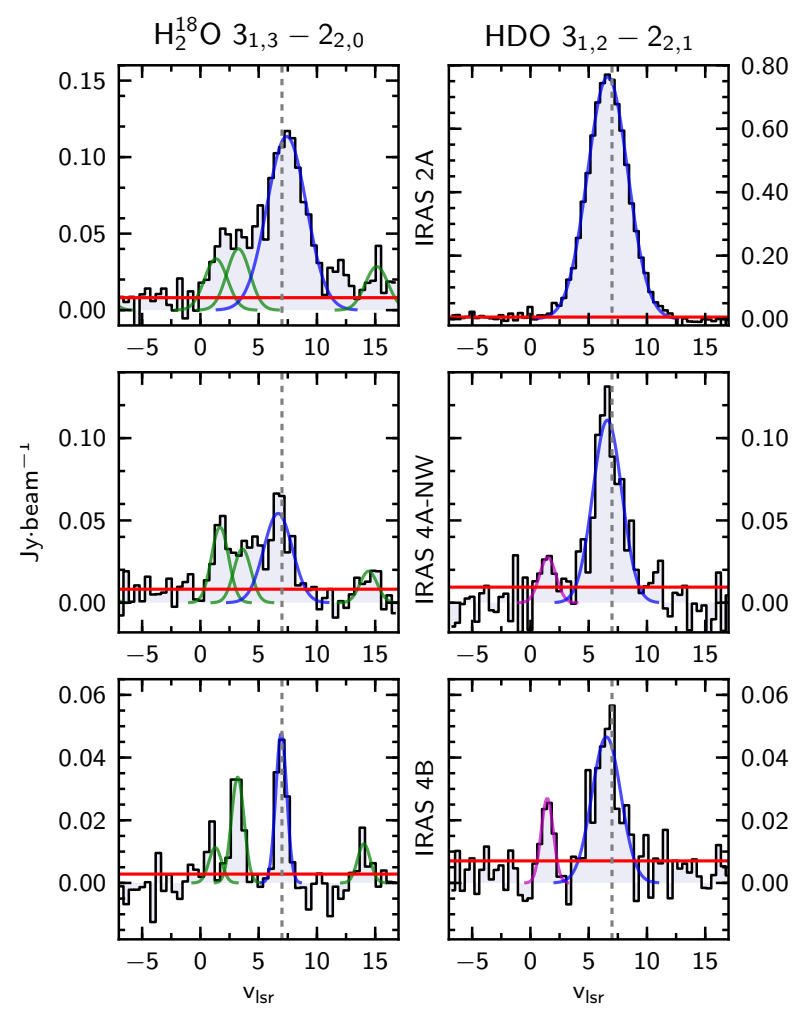

Fig. 1. Continuum subtracted spectra of the $\mathrm{H}_{2}^{18} \mathrm{O} \quad 3_{1,3}-2_{2,0}$ and HDO $3_{1,2}-2_{2,1}$ lines toward the three sources NGC-1333 IRAS 2A, IRAS 4A-NW, and IRAS 4B. The $\mathrm{H}_{2}^{18} \mathrm{O}$ spectra are from Jørgensen \& van Dishoeck (2010a) and Persson et al. (2012). The green lines shows the $\mathrm{CH}_{3} \mathrm{OCH}_{3}$, and blue $\mathrm{H}_{2}^{18} \mathrm{O}$. In some of the HDO spectra (bottom two), a second line can be seen (magenta) at $1.5 \mathrm{~km} \mathrm{~s}^{-1}$, this is from $\mathrm{CH}_{3} \mathrm{OCHO}$. The parental cloud velocity is shown by the dotted line $\left(v_{\mathrm{lsr}}=7 \mathrm{~km} \mathrm{~s}^{-1}\right)$ and the Gaussian fits (green, magenta, and blue) to the lines are plotted along with the RMS (red). We note the different scales of the spectra toward IRAS $2 \mathrm{~A}$.

queried through the Splatalogue ${ }^{4}$ interface. The molecular parameters for the lines are tabulated in Table A.2. The continuum subtracted spectra of both the $\mathrm{H}_{2}^{18} \mathrm{O} 3_{1,3}-2_{2,0}$ and the HDO $3_{1,2}-2_{2,1}$ transition extracted toward the continuum peak for all the sources (excluding IRAS 4A-SE) are shown in Fig. 1 $\left(\mathrm{H}_{2}^{18} \mathrm{O}\right.$ spectra from Jørgensen \& van Dishoeck 2010a; Persson et al. 2012). Figure 2 shows the spectra for the HDO $3_{1,2}-2_{2,1}$ and $2_{1,1}-2_{1,2}$ transitions toward IRAS 2 A. All lines used in this analysis are detected at high signal-to-noise (i.e. $\gtrsim 10 \sigma$ ). In the $\mathrm{H}_{2}^{18} \mathrm{O}$ spectra, the lines seen besides water are due to dimethyl ether $\left(\mathrm{CH}_{3} \mathrm{OCH}_{3}\right)$, for more details and information about the line assignment see Jørgensen \& van Dishoeck (2010a); Persson et al. (2012). In the new HDO spectra for the $3_{1,2}-2_{2,1}$ transition,

4 http://splatalogue.net 

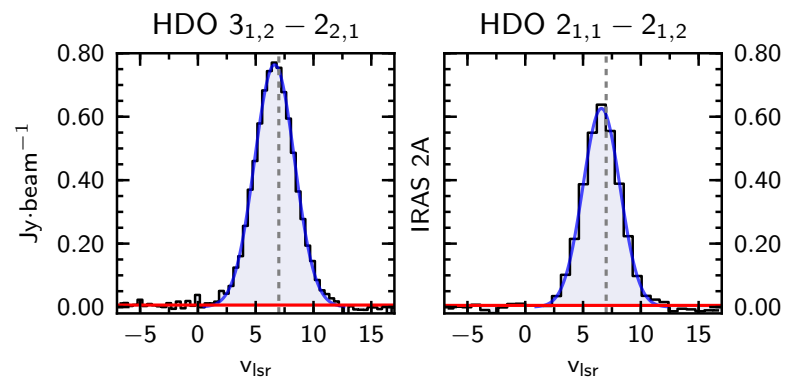

Fig. 2. Continuum subtracted spectra of the HDO $3_{1,2}-2_{2,1}(225 \mathrm{GHz})$ and $2_{1,1}-2_{1,2}(241 \mathrm{GHz})$ lines toward IRAS2A. The parental cloud velocity is shown by the dotted line $\left(v_{\mathrm{lsr}}=7 \mathrm{~km} \mathrm{~s}^{-1}\right)$ and the Gaussian fits (blue) to the HDO lines are plotted along with the RMS (red).

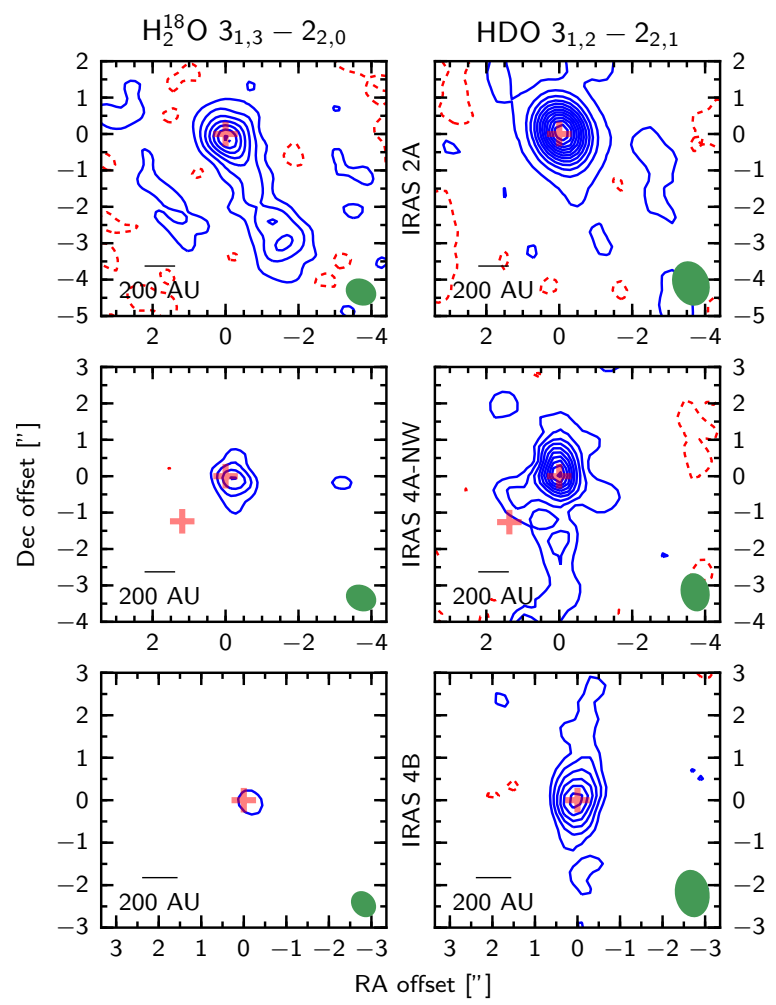

Fig. 3. Integrated intensity maps for the HDO $3_{1,1}-3_{3,1}$ and $\mathrm{H}_{2}^{18} \mathrm{O}$ $3_{1,3}-2_{2,0}$ in the interval $v_{\text {line }} \pm F W H M$ toward all three sources. The beam is shown in the lower right corner and the red crosses show the position of continuum peaks. Contours start at $41.1 \mathrm{mJy} \mathrm{km} \mathrm{s}^{-1}$ ( $5 \sigma$ steps for $\mathrm{H}_{2}^{18} \mathrm{O}$ and $20 \sigma$ for HDO) for IRAS $2 \mathrm{~A}, 50.1 \mathrm{mJy} \mathrm{km} \mathrm{s}^{-1}$ ( $2 \sigma$ steps) for IRAS 4A, and $29.8 \mathrm{mJy} \mathrm{km} \mathrm{s}^{-1}$ ( $2 \sigma$ steps) for IRAS 4B, dashed contours represent negative values.

at $v_{\mathrm{lsr}} \approx 1.5 \mathrm{~km} \mathrm{~s}^{-1}$ a line from methyl formate $\left(\mathrm{CH}_{3} \mathrm{OCHO}\right.$, $E_{\mathrm{u}}=36.3 \mathrm{~K}$ ) is seen toward IRAS 4A and IRAS 4B (Fig. 1). The targeted HDO line is strong in all the sources. Just as with the $\mathrm{H}_{2}^{18} \mathrm{O}$ line, no $\mathrm{HDO}$ is detected toward IRAS 4A-SE.

As noted in Jørgensen \& van Dishoeck (2010a) and Persson et al. $(2012,2013)$ the $\mathrm{H}_{2}^{18} \mathrm{O}$ lines are not found to be masing in these sources on the observed scales. This is in contrast to the $3_{1,3}-2_{2,0}$ transition of the main isotopologue, $\mathrm{H}_{2}^{16} \mathrm{O}$, which is masing in many sources on single-dish scales (Cernicharo et al. 1994). The narrow widths of all detected $\mathrm{H}_{2}^{18} \mathrm{O}$ and HDO lines presented here demonstrate that the lines do not originate from any outflow in these sources, where the lines have been observed to have broader widths (i.e., $>5 \mathrm{~km} \mathrm{~s}^{-1}$ Kristensen et al. 2010).

Figure 3 shows the continuum subtracted intensity maps for all three sources and water lines. For IRAS 2A Fig. 4 shows the

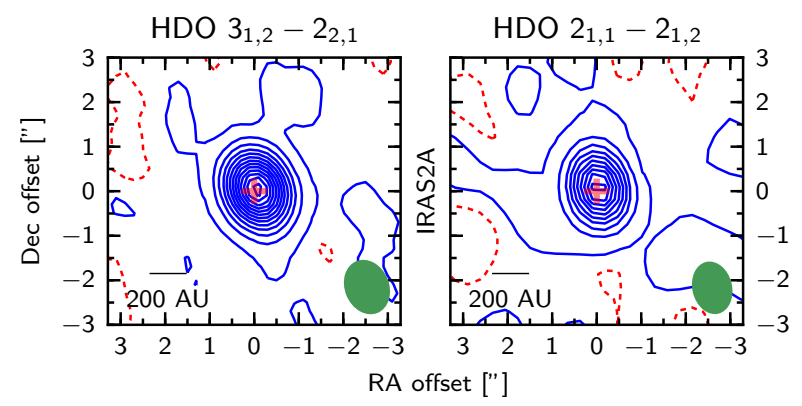

Fig. 4. Integrated intensity maps for the $3_{1,2}-2_{2,1}(225 \mathrm{GHz})$ and $2_{1,1}-2_{1,2}(241 \mathrm{GHz})$ transitions of HDO toward IRAS 2A, in the interval $v_{\text {line }} \pm F W H M$. The beam is shown in the lower right corner and the red crosses show the position of continuum peaks. Contours start at $39.6 \mathrm{mJy} \mathrm{km} \mathrm{s}^{-1}$ (steps of $20 \sigma$ ), dashed contours represent negative values.

Table 3. Results from fits to the spectra and the $(u, v)$-plane.

\begin{tabular}{|c|c|c|c|}
\hline Source & $\begin{array}{l}\text { Intensity } \\
{\left[\mathrm{Jy} \mathrm{km} \mathrm{s}^{-1}\right]}\end{array}$ & $\begin{array}{l}F W H M \\
{\left[\mathrm{~km} \mathrm{~s}^{-1}\right]}\end{array}$ & $\begin{array}{l}\text { Size }^{a} \\
{[\operatorname{arcsec}]}\end{array}$ \\
\hline $\begin{array}{l}\text { I2A } \\
\text { I4A-NW } \\
\text { I4B }\end{array}$ & $\begin{array}{l}3.98 \pm 0.80 \\
1.97 \pm 0.39 \\
0.36 \pm 0.07 \\
\end{array}$ & $\begin{array}{l}\text { HDO } 3_{1,2}-2_{2,1} \\
4.1 \pm 0.1 \\
3.7 \pm 0.2 \\
2.5 \pm 0.2\end{array}$ & $\begin{array}{l}0.4 \pm 0.1 \\
1^{\prime \prime} 6 \pm 0.1 \\
0.5 \pm 0.1 \\
\end{array}$ \\
\hline $\mathrm{I} 2 \mathrm{~A}$ & $3.88 \pm 0.78$ & $\begin{array}{l}\text { HDO } 2_{1,1}-2_{1,2} \\
4.0 \pm 0.1\end{array}$ & $0.5 \pm 0.1$ \\
\hline $\begin{array}{l}\text { I2A } \\
\text { I4A-NW } \\
\text { I4B }\end{array}$ & $\begin{array}{l}0.98 \pm 0.2 \\
0.27 \pm 0.05 \\
0.09 \pm 0.02 \\
\end{array}$ & $\begin{array}{l}\mathrm{H}_{2}^{18} \mathrm{O} 3_{1,3}-2_{2,0} \\
4.0 \pm 0.2 \\
2.9 \pm 0.3 \\
0.9 \pm 0.1\end{array}$ & $\begin{array}{l}0 . \\
0 . \\
0.6 \pm 0.1 \\
0 . \\
0.2 \pm 0.1 \\
\end{array}$ \\
\hline
\end{tabular}

Notes. The errors in the FWHM and size are from the statistical fits, while the uncertainty in intensity is assumed to be 20\%. ${ }^{(a)}$ FWHM of circular Gaussian fit in the $(u, v)$-plane.

additional HDO line at $241.6 \mathrm{GHz}$ together with the $225.9 \mathrm{GHz}$ line. The intensity maps for each source are plotted on the same scale, i.e., the contour lines indicate the same intensities.

Circular Gaussian profiles were fitted to the emission of each line in the $(u, v)$-plane after integrating over channels corresponding to $v_{\mathrm{lsr}} \pm F W H M$. Table 3 lists the FWHM of the 1D Gaussian fitted in the spectra, and size and intensity from fits in the $(u, v)$ plane of the integrated intensity for all the lines. The line widths roughly agree between the lines, being slightly wider in the HDO spectra (see Table 3).

\section{Analysis}

\subsection{Spectra and maps}

The spectra show similar characteristics for the observed sources and lines. The emission is compact and traces the warm water within $150 \mathrm{AU}$ of the central sources. The integrated map for the IRAS 4A protobinary shows extended emission, partly aligned with the southern, blue-shifted outflow (Jørgensen et al. 2007). In contrast, the south-west outflow of IRAS $2 \mathrm{~A}$ seen in $\mathrm{H}_{2}^{18} \mathrm{O}$ is not detected in either of the two HDO lines. The ratio between the HDO line(s) and the $\mathrm{H}_{2}^{18} \mathrm{O}$ line is similar for IRAS $2 \mathrm{~A}$ and IRAS $4 \mathrm{~B}$, with HDO showing stronger emission than that of $\mathrm{H}_{2}^{18} \mathrm{O}$ by a factor of four. In IRAS 4A-NW, the HDO $3_{1,2}-2_{2,1}$ line is even brighter - about seven times stronger than the $\mathrm{H}_{2}^{18} \mathrm{O}$ line. Methyl formate $\left(\mathrm{CH}_{3} \mathrm{OCHO}\right)$ is clearly seen toward IRAS 4A-NW and IRAS $4 \mathrm{~B}$ in the $225 \mathrm{GHz}$ spectra, while it is absent in the spectrum toward IRAS 2A. All lines toward IRAS 4B show very compact emission. 


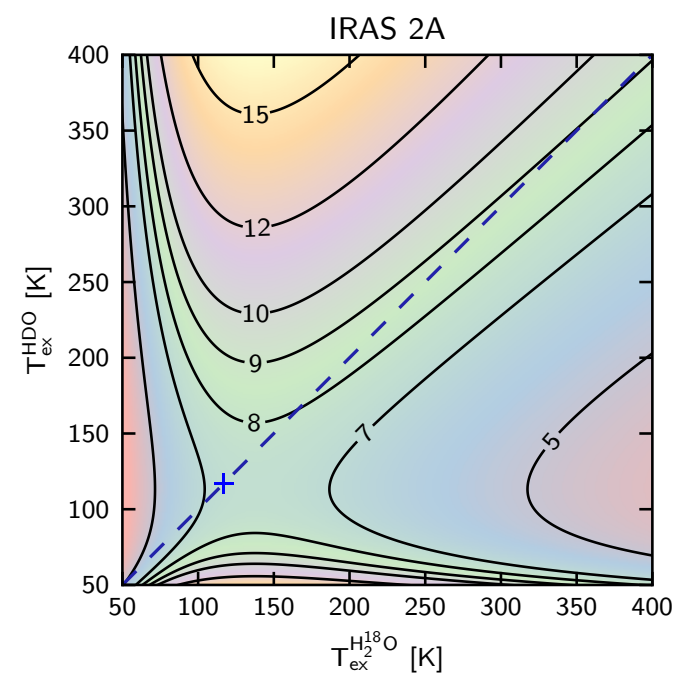

Fig. 5. Variation of the water deuterium fractionation $\left(\mathrm{HDO} / \mathrm{H}_{2} \mathrm{O}\right)$ as a function of excitation temperature $(40-400 \mathrm{~K})$ for the observed water isotopologues. Contour values are $\times 10^{-4}$ and the dashed blue line shows where $T_{\mathrm{ex}}^{\mathrm{H}_{2}^{18} \mathrm{O}}=T_{\mathrm{ex}}^{\mathrm{HDO}}$, and the cross the value determined for IRAS 2A, i.e. at $T_{\mathrm{ex}}=124$.

\subsection{Column densities, excitation temperature and the $\mathrm{HDO} / \mathrm{H}_{2} \mathrm{O}$ ratio}

With two transitions of HDO observed toward IRAS 2A with different upper energy levels $\left(E_{\mathrm{u}}\right)$, a rough estimate of the excitation temperature $\left(T_{\mathrm{ex}}\right)$ can be made. One issue is that the energy levels are quite close (167 vs. $95 \mathrm{~K})$, which increases the uncertainty of the determined excitation temperature. Assuming that the HDO is optically thin and in LTE, and that the emission fills the beams uniformly, the excitation temperature for HDO in IRAS $2 \mathrm{~A}$ is $T_{\mathrm{ex}}=124 \pm 60 \mathrm{~K}$, using a $20 \%$ flux calibration uncertainty. This excitation temperature reflects the bulk temperature of the medium averaged over the entire synthesized beam.

We can estimate the column density of HDO over the observed beam in all of the sources by assuming LTE with $T_{\mathrm{ex}}=$ $124 \mathrm{~K}$ as determined for HDO in IRAS 2A, that the mission fills the beam and is optically thin. For IRAS $2 \mathrm{~A}$ this is $11.9 \pm 2.4 \times$ $10^{15} \mathrm{~cm}^{-2}$, for IRAS $4 \mathrm{~A}-\mathrm{NW}$ it is $8.8 \pm 1.8 \times 10^{15} \mathrm{~cm}^{-2}$, and for IRAS 4B $1.5 \pm 0.3 \times 10^{15} \mathrm{~cm}^{-2}$. Assuming $T_{\mathrm{ex}}=124 \mathrm{~K}$ for the $\mathrm{H}_{2}^{18} \mathrm{O}$ line as well gives column densities for the three sources consistent with the results of Persson et al. (2012), where $T_{\mathrm{ex}}=170 \mathrm{~K}$ was assumed.

With the column density of both HDO and $\mathrm{H}_{2}^{18} \mathrm{O}$, we deduce the water deuterium fractionation in all three protostars. For IRAS $2 \mathrm{~A}$ the $\mathrm{HDO} / \mathrm{H}_{2} \mathrm{O}$ ratio is then $7.4 \pm 2.1 \times 10^{-4}$, for IRAS $4 \mathrm{~A}-\mathrm{NW}$ it is $19.1 \pm 5.4 \times 10^{-4}$ and for IRAS $4 \mathrm{~B}$ it is $5.9 \pm 1.7 \times 10^{-4}$. In the calculations a ${ }^{16} \mathrm{O} /{ }^{18} \mathrm{O}$ ratio of 560 was assumed, appropriate for the local interstellar medium (ISM; Wilson \& Rood 1994). For IRAS 4B, the deduced $\mathrm{HDO} / \mathrm{H}_{2} \mathrm{O}$ ratio agrees within the uncertainties with the upper limit derived by Jørgensen \& van Dishoeck (2010b) using lower sensitivity SMA data.

To investigate the effects of different excitation temperatures, Fig. 5 shows the $\mathrm{HDO} / \mathrm{H}_{2} \mathrm{O}$ ratio in IRAS $2 \mathrm{~A}$ in the $T_{\mathrm{ex}}$ range $40-400 \mathrm{~K}$. As can be seen in the figure, the $\mathrm{HDO} / \mathrm{H}_{2} \mathrm{O}$ ratio does not change significantly over this wide range of excitation temperatures: even if $T_{\mathrm{ex}}\left(\mathrm{H}_{2}^{18} \mathrm{O}\right)$ is significantly different from $T_{\mathrm{ex}}(\mathrm{HDO})$, the derived abundance ratios only vary by a factor of 3 from $5-15 \times 10^{-4}$.

\subsection{Radiative transfer modeling}

To test our assumption of optically thin lines used to derive column densities in Sect. 4.2, full radiative transfer models of spherically symmetric envelopes have been run. These same type of models have been used to interpret the water and HDO emission lines observed by Herschel and single-dish ground based telescopes (Coutens et al. 2012, 2013). In these models, the molecular excitation is computed at each position in the envelope and subsequent line radiative transfer is performed. The interferometric data originate in the inner $\sim 100 \mathrm{AU}$ which is the scale on which the physical models are not well constrained. Previous observations have shown that flattened disk-like structures appear on scales $\$ 300$ AU (Jørgensen et al. 2005; Chiang et al. 2012). Because of the lack of proper models, we adopt the spherically symmetric structure to assess the validity of the optically thin LTE approximation. Radiative transfer models were run for two cases: IRAS 16293-2422 and IRAS 2A.

The physical models start with a density structure which follows a power-law structure, as specified below for each of the sources. The dust temperature is then computed self-consistently as function of radius in the envelope for a given luminosity using the 1-D spherical dust continuum radiative transfer code TRANSPHERE (Dullemond et al. 2002). For the typical densities of the protostellar envelopes the gas is expected to be coupled to the dust and we thus assume that the gas and dust temperatures are identical (Jørgensen et al. 2002). The resulting temperature and density structure are then used as input to the Monte Carlo radiative transfer code RATRAN (Hogerheijde \& van der Tak 2000). Since the line profiles do not show any complicated velocity structures the models only include a doppler broadening parameter (the "doppler-b parameter" $0.6 \times F W H M$ ). The outer radius is defined as where $T_{\text {dust }}=10 \mathrm{~K}$ or $n=10^{4} \mathrm{~cm}^{-3}$, whichever comes first. HDO collisional rate coefficients of Faure et al. (2012) are used to calculate non-LTE population levels (LAMBDA database, Schöier et al. 2005). To produce the collisional rate coefficients for para- $\mathrm{H}_{2}^{18} \mathrm{O}$, the rates for $\mathrm{H}_{2}^{16} \mathrm{O}$ from Daniel et al. (2011) for $5<T<1500 \mathrm{~K}$ were combined with the tabulated levels for para- $\mathrm{H}_{2}^{18} \mathrm{O}$ from the JPL catalog (Pickett et al. 1998). The abundance of $\mathrm{HDO}$ or $\mathrm{p}-\mathrm{H}_{2}^{18} \mathrm{O}$ is then varied to obtain the best fit to the observed lines.

To determine the $\mathrm{H}_{2} \mathrm{O}$ abundance, the best-fit $\mathrm{p}-\mathrm{H}_{2}^{18} \mathrm{O}$ abundance has to be multiplied with the fraction of para to total water ratio $(4$, i.e. $o / p=3 / 1)$ and the ${ }^{16} \mathrm{O} /{ }^{18} \mathrm{O}$ ratio $(560$, Wilson $\&$ Rood 1994). The molecular abundance is assumed to follow a "jump" abundance profile (e.g., Schöier et al. 2004), with an inner and outer abundance that changes discontinuously at the point in the envelope where $T_{\text {dust }}=100 \mathrm{~K}$, i.e., where water evaporates off the dust grains (Fraser et al. 2001). The radiative transfer model gives as output a model image cube with units Jy/pixel; this is then convolved with a beam corresponding to the observations and the integrated intensity is compared to those observed in Table 3 and Persson et al. (2013). To check where the emission in the relevant lines originates, the excitation is first solved for a model that covers the entire envelope, i.e. out to $r_{\text {out }}$. Then an increasing number of cells are included in the ray-tracer, and the resulting integrated intensities are compared. This shows that the majority $(>97 \%)$ of the emission in the observed lines comes from the region where $T \geq 100 \mathrm{~K}$. To increase the numerical convergence, the envelope model for IRAS $2 \mathrm{~A}$ was truncated at relatively small radii (6100 AU). The less dense and cold outer parts $(R>6100 \mathrm{AU})$ of the envelope does not contribute significantly to any absorption or emission of the observed lines. 
For the IRAS 16293-2422 envelope, we adopt the same physical structure as developed by Crimier et al. (2010), which also allows us to compare our results to Coutens et al. (2012). In the original model a velocity field describing infall was used, here we set the turbulence doppler-b parameter to $1 \mathrm{~km} \mathrm{~s}^{-1}$ because we are only interested in fitting the total integrated intensity. Unless the lines are significantly optically thick, the adopted doppler-b parameter will only affect the line profile and not the total integrated intensity. The density structure of the envelope is described by Shu (1977) as

$$
\begin{aligned}
& n(r)=n\left(r_{0}\right)\left(\frac{r}{r_{0}}\right)^{-1.5} \quad r \leq r_{\text {inf }} \\
& n(r)=n\left(r_{0}\right)\left(\frac{r}{r_{0}}\right)^{-2} \quad r>r_{\mathrm{inf}}
\end{aligned}
$$

where $r_{\text {inf }}=1280 \mathrm{AU}, r_{0}=76 \mathrm{AU}$ and $n\left(r_{0}\right)=2 \times 10^{8} \mathrm{~cm}^{-3}$ (the $\mathrm{H}_{2}$ number density at $r_{0}$ where $T_{\text {dust }}=100 \mathrm{~K}$ ). The dust opacity as a function of the frequency is set to a power-law emissivity model, i.e.,

$\kappa=\kappa_{0}\left(\frac{v}{v_{0}}\right)^{\beta}$

where $\beta=1.8, \kappa=15 \mathrm{~cm}^{2} \mathrm{~g}_{\text {dust }}^{-1}$ and $v_{0}=10^{12} \mathrm{~Hz}$. This dust opacity relation was adopted by Coutens et al. (2012) in order to fit the continuum observed with the Herschel-HIFI from 0.5 to $1 \mathrm{THz}$. The cold outer abundance at $T<100 \mathrm{~K}$ is fixed to $X_{\text {out }}=3 \times 10^{-11}$ as found by Coutens et al. (2012) for both HDO and $\mathrm{H}_{2}^{18} \mathrm{O}$ with respect to $\mathrm{H}_{2}$. Changing it within a reasonable interval $\left( \pm 10 \times X_{\text {out }}\right)$ does not change the derived warm inner abundances significantly.

The best fit to the $203 \mathrm{GHz} \mathrm{H} \mathrm{H}_{2}^{18} \mathrm{O}$ line of IRAS 162932422 requires an inner abundance of $X_{\text {in }}=4.5 \pm 0.9 \times 10^{-8}$ $\left(1.0 \pm 0.2 \times 10^{-4}\right.$ for $\left.\mathrm{H}_{2} \mathrm{O}\right)$, and for the HDO line at $225.9 \mathrm{GHz}$ requires $X_{\text {in }}=11.0 \pm 2.2 \times 10^{-8}$. These abundances result in a $\mathrm{HDO} / \mathrm{H}_{2} \mathrm{O}$ ratio of $10.9 \pm 3.1 \times 10^{-4}$. The models show that both HDO lines at $225.9 \mathrm{GHz}$ and $241.6 \mathrm{GHz}$ are marginally optically thick whereas the $\mathrm{H}_{2}^{18} \mathrm{O}$ line is neither masing nor optically thick. The derived abundance ratio clearly agrees with the previously stated LTE, optically thin value of $9.2 \pm 2.6 \times 10^{-4}$ within the uncertainties (Persson et al. 2013). If the o/p ratio for the main collision partner $\left(\mathrm{H}_{2}\right)$ is changed to 0 , the $\mathrm{HDO} / \mathrm{H}_{2} \mathrm{O}$ ratio goes down by $20 \%$. For water, if an o/p ratio $\left(\mathrm{H}_{2} \mathrm{O}\right)$ of 1 and a ${ }^{16} \mathrm{O} /{ }^{18} \mathrm{O}$ ratio of 400 is assumed the $\mathrm{HDO} / \mathrm{H}_{2} \mathrm{O}$ ratio increases by a factor of 2.8 . This shows that the assumed $\mathrm{o} / \mathrm{p}$ ratio of either $\mathrm{H}_{2} \mathrm{O}$ or $\mathrm{H}_{2}$ and the ${ }^{16} \mathrm{O} /{ }^{18} \mathrm{O}$ ratio can affect the resulting $\mathrm{HDO} / \mathrm{H}_{2} \mathrm{O}$ ratio down by $20 \%$ and up by actor of 2.8 .

For IRAS $2 \mathrm{~A}$ we have used the model described by Kristensen et al. (2012) and it extends from 35.9 AU (terminates where $T>250 \mathrm{~K}$ ) to $17950 \mathrm{AU}$ (but truncated at $6100 \mathrm{AU}$ ). The density profile is a single power-law, $n(r)=n\left(r_{0}\right)\left(r / r_{0}\right)^{-1.7}$, where $r_{0}=r_{\text {in }}$ and $n\left(r_{0}\right)=4.9 \times 10^{8} \mathrm{~cm}^{-3}$. The dust opacity is set to Table 1, Col. 5 in Ossenkopf \& Henning (1994) (e.g., OH5 - dust grains with thin ice mantles) and the dust temperature is again computed self-consistently. The outer abundance is fixed to $X_{\text {out }}=3 \times 10^{-11}$ for both HDO and $\mathrm{H}_{2}^{18} \mathrm{O}$, and as for IRAS 16293-2422, changing it within a reasonable interval $( \pm 10 \times)$ does not change the derived abundances significantly. The best fit inner abundance for the $\mathrm{H}_{2}^{18} \mathrm{O}$ line is $X_{\text {in }}=4.8 \pm 1.0 \times 10^{-8}\left(1.1 \pm 0.2 \times 10^{-4}\right.$ for $\left.\mathrm{H}_{2} \mathrm{O}\right)$, and $X_{\text {in }}=34.0 \pm 6.8 \times 10^{-8}$ for the HDO line at $225.9 \mathrm{GHz}$. This corresponds to a $\mathrm{HDO} / \mathrm{H}_{2} \mathrm{O}$ ratio of $31.6 \pm 8.9 \times 10^{-4}$. While the HDO abundance derived from the $225 \mathrm{GHz}$ line also fits the $241 \mathrm{GHz}$ line within the uncertainties, the $143 \mathrm{GHz}$ line observed by Taquet et al. (2013) is fit by a somewhat lower HDO abundance of $X_{\mathrm{in}}=24.3 \pm 4.9$ giving a $\mathrm{HDO} / \mathrm{H}_{2} \mathrm{O}$ ratio of $22.3 \pm 6.4 \times 10^{-4}$. All these $\mathrm{HDO}$ lines give a $\mathrm{HDO} / \mathrm{H}_{2} \mathrm{O}$ ratio that is higher by a factor of $3-4$ than the optically thin LTE value of $7.4 \pm 2.1 \times 10^{-4}$. This difference is largely due to the optical depth effects of the HDO lines.

The models for both IRAS 16293-2422 and IRAS 2A indicate that the HDO lines are marginally optically thick. This could indicate that the derived HDO column densities from the optically thin calculation (in Sect. 4.2) are slightly underestimated for all four sources. On the other hand, the $\mathrm{H}_{2}^{18} \mathrm{O}$ line at $203.4 \mathrm{GHz}$ is well constrained by LTE and is optically thin. Combined, this would imply an underestimate of the $\mathrm{HDO} / \mathrm{H}_{2} \mathrm{O}$ ratio. However, the models show that this effect is minor, a factor of $\sim 3$ or less based on a comparison of the ratios derived from the radiative transfer models and the direct LTE estimates. The IRAS 16293-2422 example also shows that differential excitation effects of $\mathrm{HDO}$ versus $\mathrm{H}_{2}^{18} \mathrm{O}$ can counteract the HDO optical depth effects.

\section{Discussion and conclusions}

All deduced $\mathrm{HDO} / \mathrm{H}_{2} \mathrm{O}$ ratios are consistent with our previous low estimates for two deeply-embedded protostars (Persson et al. 2013; Jørgensen \& van Dishoeck 2010b). The consistently low $\mathrm{HDO} / \mathrm{H}_{2} \mathrm{O}$ ratios for all four sources suggests that the chemical evolution of water for these sources is similar.

Our high-resolution observations make it possible to focus on the compact warm gas. The sources show some extended emission in $\mathrm{HDO}$ and/or $\mathrm{H}_{2}^{18} \mathrm{O}$ that would be included in any lower-resolution (single-dish) observations. Another key to our conclusions is that the observed lines are optically thin or at most marginally optically thick. Because of their higher Einstein A coefficients, the $3_{1,2}-3_{0,3}$ lines of $\mathrm{H}_{2}^{16} \mathrm{O}$ and $\mathrm{H}_{2}^{18} \mathrm{O}$ observed with Herschel rapidly become optically thick, each at slightly different radii complicating the analysis (Visser et al. 2013). Unless the optical depth is properly treated, the $\mathrm{HDO} / \mathrm{H}_{2} \mathrm{O}$ ratios deduced using Herschel data are likely to be overestimated. The ratio estimated for IRAS $2 \mathrm{~A}$ by Visser et al. accounting for the optical thickness of the Herschel lines gives a $\mathrm{HDO} / \mathrm{H}_{2} \mathrm{O}$ ratio of $1 \times 10^{-3}$, in agreement with our estimates.

The recently deduced warm $\mathrm{HDO} / \mathrm{H}_{2} \mathrm{O}$ ratios for IRAS $2 \mathrm{~A}$ $\left(3 \times 10^{-3}\right)$ and IRAS $4 \mathrm{~A}\left(5 \times 10^{-3}\right)$ at the relevant densities $\left(n_{\mathrm{H}_{2}}=\right.$ $1 \times 10^{8} \mathrm{~cm}^{-3}$ ) by Taquet et al. (2013) are factors of 3-5 higher than our estimates. With a lower spectral (4 vs. $\left.0.1 \mathrm{~km} \mathrm{~s}^{-1}\right)$ and spatial ( $2^{\prime \prime}$ vs. $\left.0.8^{\prime \prime}\right)$ resolution than that of our $\mathrm{H}_{2}^{18} \mathrm{O}$ data, the HDO observations used by Taquet et al. probe larger scales, thus are affected by extended emissions as observed in our observations at scales $\gtrsim 500 \mathrm{AU}$ (with higher $\mathrm{HDO} / \mathrm{H}_{2} \mathrm{O}$ ratios as a probable result). Furthermore, the upper energy levels of the HDO and $\mathrm{H}_{2}^{18} \mathrm{O}$ transitions differ significantly, $E_{\mathrm{u}}=319 \mathrm{~K}$ for the HDO $4_{2,2}-4_{2,3}$ transition vs. $204 \mathrm{~K}$ for the $\mathrm{H}_{2}^{18} \mathrm{O} 3_{1,3}-2_{2,0}$. The transitions could originate in slightly different regions depending on the source structure.

Coutens et al. (2013) used Herschel and several groundbased single dish telescopes to observe lines of HDO toward IRAS 4A and IRAS 4B. From 1D radiative transfer models and using the $\mathrm{H}_{2}^{18} \mathrm{O}$ column density by Persson et al. (2012), they estimated $\mathrm{HDO} / \mathrm{H}_{2} \mathrm{O}$ ratios in the inner warm regions of IRAS $4 \mathrm{~A}$ $\left(4.0-30.0 \times 10^{-4}\right)$ and IRAS 4B $\left(1.0-37.0 \pm 10^{-4}\right)$. To reproduce the absorption seen in some of the spectral lines, a cold foreground absorbing layer is introduced. The results for the warm 
M. V. Persson et al.: The deuterium fractionation of water on solar-system scales in deeply-embedded low-mass protostars

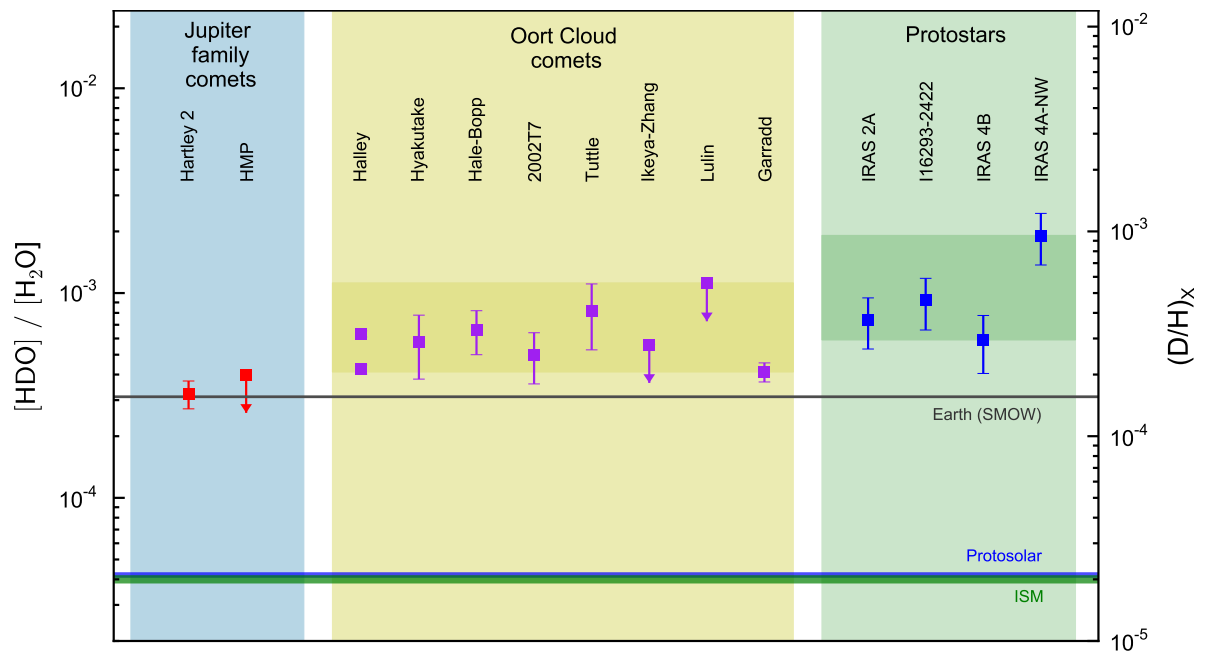

Fig. 6. Values of the $\mathrm{HDO} / \mathrm{H}_{2} \mathrm{O}$ ratio, for different objects. For references, see Table A.1. Error bars for protostars reflect those obtained using the optically thin LTE approximation.

$\mathrm{HDO} / \mathrm{H}_{2} \mathrm{O}$ ratio show a wide range but are consistent with our findings, namely that the ratio is similar for the two protostars, and that it is at most a few $\times 10^{-3}$.

The radiative transfer modeling highlights the uncertainties involved in the derivation of the $\mathrm{HDO} / \mathrm{H}_{2} \mathrm{O}$ ratio. However, the ratios deduced with the optically thin LTE model-independent (i.e., not employing physical models and radiative transfer calculations to interpret the emission) method used in this paper and in Persson et al. (2013) is direct, and agrees with the full modeling within a factor of 3-4. Until the physical structure on small scales can be constrained, running full radiative transfer models can not yield a more accurate $\mathrm{HDO} / \mathrm{H}_{2} \mathrm{O}$ ratio in the warm gas. However, spherically symmetric radiative transfer modeling can give indications on non-LTE effects and is a valid approximation for the outer envelope (e.g., Mottram et al. 2013). Furthermore, the modeling also confirms that the observed transitions of the water isotopologues are not masing in these environments.

The emerging picture of the water deuterium fractionation in warm inner regions of deeply-embedded low-mass protostars is therefore that of a consistent and relatively low ratio. While the protostars in this study are all located in the same star forming cloud, NGC 1333, IRAS 16293-2422 in Persson et al. (2013) is located in a different cloud, $\rho$ Ophiuchus. Its similar $\mathrm{HDO} / \mathrm{H}_{2} \mathrm{O}$ ratio indicates that a different birth environment does not necessarily imply a different ratio. To confirm this, $\mathrm{HDO} / \mathrm{H}_{2} \mathrm{O}$ ratios determination toward more sources are needed.

The infall timescales for the early, deeply-embedded stages are generally too short for any significant gas-phase chemical processing of water to take place in the warm $>100 \mathrm{~K}$ gas before the material enters the disk (Schöier et al. 2002). Therefore the gaseous water observed here probably originates by sublimation of ice formed earlier in the evolution.

In Fig. 6 the deuterium fractionation of water for all four low-mass protostars (IRAS 2A, IRAS 4A-NW, IRAS 4B and IRAS 16293-2422) is presented together with those found in comets, Earth, ISM and protosolar values. The small differences in the amount of deuteration seen toward the inner regions of protostars and the comets in our solar system suggests that only small amounts of processing of water takes place between the deeply embedded stages and the formation of comets and planetesimals. If water was delivered to Earth by comets and small solar system bodies during the early evolution of the Sun, this would be the same water that was formed directly on the grain surface in the cold early stages of the formation of our Sun.

\section{Summary and outlook}

In this paper high-resolution interferometric observations of the HDO $3_{1,2}-2_{2,1}$ line at $225.9 \mathrm{GHz}$ toward three deeply embedded protostars have been presented. In addition, the HDO $2_{1,1}-2_{1,2}$ line was observed toward one of the sources. With previous observations of $\mathrm{H}_{2}^{18} \mathrm{O}$ (Persson et al. 2012), we deduce a model independent $\mathrm{HDO} / \mathrm{H}_{2} \mathrm{O}$ ratio.

- Observations of two HDO lines at high angular resolution give an excitation temperature of $T_{\mathrm{ex}}=124 \pm 60 \mathrm{~K}$ for IRAS 2A.

- Assuming that the water emission is optically thin and is in LTE at $T_{\mathrm{ex}}=124 \mathrm{~K}$ for all three sources, we derive a $\mathrm{HDO} / \mathrm{H}_{2} \mathrm{O}$ ratio of $7.4 \pm 2.1 \times 10^{-4}$ for IRAS $2 \mathrm{~A}$, $19.1 \pm 5.4 \times 10^{-4}$ for IRAS $4 \mathrm{~A}-\mathrm{NW}$ and $5.9 \pm 1.7 \times 10^{-4}$ for IRAS $4 \mathrm{~B}$.

- The high-resolution interferometric observations give $\mathrm{HDO} / \mathrm{H}_{2} \mathrm{O}$ ratios in a model independent way with an accuracy of a factor of 3-4, as confirmed by using non-LTE radiative transfer models. The fact that the observed lines have much lower optical depths than those observed with Herschel explains much of the early discrepancies in inferred values.

- The $\mathrm{HDO} / \mathrm{H}_{2} \mathrm{O}$ ratios deduced for these deeply-embedded protostars using interferometric observations are consistent from source to source. The ratios range from 5.9 to $19.1 \times$ $10^{-4}$, close to those observed in solar system comets originating in the Oort cloud. The small difference between these reservoirs indicates little processing of material has taken place between the deeply-embedded, collapsing stage and the formation of comets/planetesimals.

Acknowledgements. We wish to thank the IRAM staff, in particular Arancha Castro-Carrizo and Chin Shin Chang, for their help with the observations and reduction of the data. We also appreciate discussions with Joe Mottram and Audrey Coutens on various aspects of water modeling. IRAM is supported by INSU/CNRS (France), MPG (Germany), and IGN (Spain). The research at Centre for Star and Planet Formation is supported by the Danish National Research Foundation and the University of Copenhagen's programme of excellence. This research was also supported by a Junior Group Leader Fellowship from the Lundbeck Foundation to J.K.J. E.v.D. acknowledge the Netherlands Organization for Scientific Research (NWO) grant 614.001.008. E.v.D. and M.V.P. acknowledge EU FP7 grant 291141 CHEMPLAN. This work has benefited from research funding from the European Community's sixth Framework Programme under RadioNet R113CT 20035058187. 
Appendix A: Tables

Table A.1. HDO/ $\mathrm{H}_{2} \mathrm{O}$ abundance ratios for different objects.

\begin{tabular}{|c|c|c|c|c|c|}
\hline Type & Object & Origin & $\begin{array}{l}\mathrm{HDO} / \mathrm{H}_{2} \mathrm{O} \\
{\left[\times 10^{-4}\right]}\end{array}$ & $\begin{array}{l}\text { Tracers } \\
\text { (Proxy) }\end{array}$ & Ref. \\
\hline Comet & 1/P Halley & (OCC) & $6.3 \pm 0.7$ & $\mathrm{H}_{3} \mathrm{O}^{+}, \mathrm{H}_{2} \mathrm{DO}^{+}$ & 1 \\
\hline Comet & C/1996 B2 Hyakutake & (OCC) & $5.8 \pm 2.0$ & $\mathrm{H}_{2}^{16} \mathrm{O}, \mathrm{HDO}$ & 2 \\
\hline Comet & C/1995 O1 Hale-Bopp & (OCC) & $6.6 \pm 1.6$ & $\mathrm{HDO}, \mathrm{OH}$ & 3 \\
\hline Comet & 8P/Tuttle & (OCC) & $8.2 \pm 2.9$ & $\mathrm{H}_{2}^{16} \mathrm{O}, \mathrm{HDO}$ & 4 \\
\hline Comet & C/2007 N3 Lulin & (OCC) & $<11.2$ & $\mathrm{H}_{2}^{16} \mathrm{O}, \mathrm{HDO}$ & 5 \\
\hline Comet & C/2002 T7 LINEAR & (OCC) & $5 \pm 1.4^{a}$ & $\mathrm{OH},{ }^{18} \mathrm{OH}, \mathrm{OD}$ & 6 \\
\hline Comet & 153P Ikeya-Zhang & (OCC) & $<5.6 \pm 0.6$ & $\mathrm{H}_{2}^{16} \mathrm{O}, \mathrm{H}_{2}^{18} \mathrm{O}, \mathrm{HDO}$ & 7 \\
\hline \multirow[t]{2}{*}{ Comet } & C/2009 P1 Garradd & (OCC) & $2.06 \pm 0.22$ & $\mathrm{H}_{2}^{18} \mathrm{O}, \mathrm{H}_{2} \mathrm{O}, \mathrm{HDO}$ & 8 \\
\hline & OCC Mean $^{c}$ & & $6.4 \pm 1.0$ & & \\
\hline Comet & 103P Hartley 2 & (JFC) & $3.2 \pm 0.5$ & $\mathrm{H}_{2}^{18} \mathrm{O}, \mathrm{HDO}$ & 9 \\
\hline Comet & 45P Honda-Mrkos-Pajdusakov (HMP) & (JFC) & $<4.0$ & $\mathrm{H}_{2}^{16} \mathrm{O}, \mathrm{H}_{2}^{18} \mathrm{O}, \mathrm{HDO}$ & 10 \\
\hline \multirow[t]{2}{*}{ Planet } & Earth (SMOW) & & $3.114 \pm 0.002$ & & 11 \\
\hline & $\begin{array}{l}\text { Protosolar } \\
\text { (L)ISM }{ }^{c}\end{array}$ & & $\begin{array}{l}0.42 \pm 0.08^{b} \\
\geq 0.40 \pm 0.02\end{array}$ & $\begin{array}{l}{ }^{3} \mathrm{He},{ }^{4} \mathrm{He} \\
\mathrm{H}, \mathrm{D}\end{array}$ & $\begin{array}{l}12 \\
13\end{array}$ \\
\hline Protostar & IRAS $16293-2422$ & $<50 \mathrm{AU}$ & $9.2 \pm 2.6$ & $\mathrm{H}_{2}^{18} \mathrm{O}, \mathrm{HDO}$ & 14 \\
\hline Protostar & & & 340 & $\mathrm{H}_{2}^{17} \mathrm{O}, \mathrm{H}_{2}^{18} \mathrm{O}, \mathrm{HD}^{18} \mathrm{O}, \mathrm{HDO}$ & 15 \\
\hline Protostar & & & 300 & $\mathrm{H}_{2}^{16} \mathrm{O}, \mathrm{HDO}$ & 16 \\
\hline Protostar & NGC 1333-IRAS 2A & $\lesssim 300 \mathrm{AU}$ & $7.4 \pm 2.1$ & $\mathrm{H}_{2}^{18} \mathrm{O}, \mathrm{HDO}$ & 17 \\
\hline Protostar & & & $\geq 100$ & $\mathrm{H}_{2}^{16} \mathrm{O}, \mathrm{HDO}$ & 18 \\
\hline Protostar & & & $30-800$ & $\mathrm{H}_{2}^{18} \mathrm{O}, \mathrm{HDO}$ & 19 \\
\hline Protostar & NGC 1333-IRAS 4A-NW & $\lesssim 300 \mathrm{AU}$ & $19.1 \pm 5.4$ & $\mathrm{H}_{2}^{18} \mathrm{O}, \mathrm{HDO}$ & 17 \\
\hline Protostar & & & $50-300$ & $\mathrm{H}_{2}^{18} \mathrm{O}, \mathrm{HDO}$ & 19 \\
\hline Protostar & & & $4-30$ & $\mathrm{H}_{2}^{18} \mathrm{O}, \mathrm{HDO}$ & 20 \\
\hline Protostar & NGC 1333-IRAS 4B & $\lesssim 300 \mathrm{AU}$ & $5.9 \pm 1.7$ & $\mathrm{H}_{2}^{18} \mathrm{O}, \mathrm{HDO}$ & 17 \\
\hline Protostar & & & $1-37$ & $\mathrm{H}_{2}^{18} \mathrm{O}, \mathrm{HDO}$ & 20 \\
\hline Protostar & & & $<6.4$ & $\mathrm{H}_{2}^{18} \mathrm{O}, \mathrm{HDO}$ & 21 \\
\hline
\end{tabular}

Notes. OCC - Oort Cloud Comet, JFC - Jupiter Family Comet. Some values are $2 \times$ the D/H ratio and that the ratio in protostars refers to the warm gas in the inner regions. ${ }^{(a)}$ Value is $2 \times \mathrm{D} / \mathrm{H} .{ }^{(b)}$ No upper limits included. ${ }^{(c)}$ Local interstellar medium, i.e. $\sim 1-2 \mathrm{kpc}$ from the Sun.

References. (1) Eberhardt et al. (1995); (2) Bockelée-Morvan et al. (1998); (3) Meier et al. (1998); (4) Villanueva et al. (2009); (5) Gibb et al. (2012); (6) Hutsemékers et al. (2008); (7) Biver et al. (2006); (8) Bockelée-Morvan et al. (2012); (9) Hartogh et al. (2011); (9) Lis et al. (2013); (11) de Laeter et al. (2003, and ref. therein); (12) Geiss \& Gloeckler (1998); Lellouch et al. (2001); (13) Prodanović et al. (2010, and ref. therein); (14) Persson et al. (2013); (15) Coutens et al. (2012); (16) Parise et al. (2005); (17) This work; (18) Liu et al. (2011); (19) Taquet et al. (2013); (20) (Coutens et al. 2013); (21) Jørgensen \& van Dishoeck (2010b).

Table A.2. Molecular data for the different lines, from JPL (Pickett et al. 1998) and CDMS (Müller et al. 2001).

\begin{tabular}{lllll}
\hline \hline Molecule & Transition & $\begin{array}{l}\text { Rest freq. } \\
{[\mathrm{GHz}]}\end{array}$ & $\begin{array}{l}E_{\mathrm{u}} \\
{[\mathrm{K}]}\end{array}$ & $\begin{array}{l}\text { Strength } \\
{\left[\log _{10} A_{\mathrm{ul}}\right]}\end{array}$ \\
\hline $\mathrm{H}_{2}^{18} \mathrm{O}$ & $3_{1,3}-2_{2,0}$ & 203.40752 & 203.7 & -5.3177 \\
$\mathrm{HDO}$ & $3_{1,2}-2_{2,1}$ & 225.89672 & 167.6 & -4.8799 \\
$\mathrm{HDO}$ & $2_{1,1}-2_{1,2}$ & 241.56155 & 95.2 & -4.9256 \\
\hline
\end{tabular}

\section{References}

Biver, N., Bockelée-Morvan, D., Crovisier, J., et al. 2006, A\&A, 449, 1255 Bockelée-Morvan, D., Gautier, D., Lis, D. C., et al. 1998, Icarus, 133, 147 Bockelée-Morvan, D., Biver, N., Swinyard, B., et al. 2012, A\&A, 544, L15 Cernicharo, J., Gonzalez-Alfonso, E., Alcolea, J., Bachiller, R., \& John, D. 1994, ApJ, 432, L59

Chiang, H.-F., Looney, L. W., \& Tobin, J. J. 2012, ApJ, 756, 168 Coutens, A., Vastel, C., Caux, E., et al. 2012, A\&A, 539, A132 Coutens, A., Vastel, C., Cabrit, S., et al. 2013, A\&A, 560, A39 Crimier, N., Ceccarelli, C., Maret, S., et al. 2010, A\&A, 519, A65 Daniel, F., Dubernet, M.-L., \& Grosjean, A. 2011, A\&A, 536, A76 de Graauw, T., Helmich, F. P., Phillips, T. G., et al. 2010, A\&A, 518, L6 de Laeter, J. R., Böhlke, J. K., De Bièvre, P., et al. 2003, Pure Appl. Chem., 75, 683

Drake, M. J. 2005, Meteor. Planet. Sci., 40, 519

Dullemond, C. P., van Zadelhoff, G. J., \& Natta, A. 2002, A\&A, 389, 464

Duncan, M., Levison, H., \& Dones, L. 2004, Comets II (Tucson: Univ. Arizona Press), 193

Eberhardt, P., Reber, M., Krankowsky, D., \& Hodges, R. R. 1995, A\&A, 302, 301

Enoch, M. L., Young, K. E., Glenn, J., et al. 2006, ApJ, 638, 293

Faure, A., Wiesenfeld, L., Scribano, Y., \& Ceccarelli, C. 2012, MNRAS, 420, 699

Fraser, H. J., Collings, M. P., McCoustra, M. R. S., \& Williams, D. A. 2001, MNRAS, 327, 1165

Geiss, J., \& Gloeckler, G. 1998, Space Sci. Rev., 84, 239

Gibb, E. L., Bonev, B. P., Villanueva, G., et al. 2012, ApJ, 750, 102

Hartogh, P., Lis, D. C., Bockelée-Morvan, D., et al. 2011, Nature, 478, 218

Hersant, F., Gautier, D., \& Huré, J.-M. 2001, ApJ, 554, 391

Hogerheijde, M. R., \& van der Tak, F. F. S. 2000, A\&A, 362, 697

Hutsemékers, D., Manfroid, J., Jehin, E., Zucconi, J.-M., \& Arpigny, C. 2008, A\&A, 490, L31

Jehin, E., Manfroid, J., Hutsemékers, D., Arpigny, C., \& Zucconi, J.-M. 2009, Earth Moon Planets, 105, 167

Jennings, R. E., Cameron, D. H. M., Cudlip, W., \& Hirst, C. J. 1987, MNRAS, 226,461

Jørgensen, J. K., \& van Dishoeck, E. F. 2010a, ApJ, 710, L72 
M. V. Persson et al.: The deuterium fractionation of water on solar-system scales in deeply-embedded low-mass protostars

Jørgensen, J. K., \& van Dishoeck, E. F. 2010b, ApJ, 725, L172

Jørgensen, J. K., Schöier, F. L., \& van Dishoeck, E. F. 2002, A\&A, 389, 908

Jørgensen, J. K., Bourke, T. L., Myers, P. C., et al. 2005, ApJ, 632, 973

Jørgensen, J. K., Harvey, P. M., Evans, II, N. J., et al. 2006, ApJ, 645, 1246

Jørgensen, J. K., Bourke, T. L., Myers, P. C., et al. 2007, ApJ, 659, 479

Kavelaars, J. J., Mousis, O., Petit, J.-M., \& Weaver, H. A. 2011, ApJ, 734, L30

Kristensen, L. E., Visser, R., van Dishoeck, E. F., et al. 2010, A\&A, 521, L30

Kristensen, L. E., van Dishoeck, E. F., Bergin, E. A., et al. 2012, A\&A, 542, A8

Lellouch, E., Bézard, B., Fouchet, T., et al. 2001, A\&A, 370, 610

Lis, D. C., Biver, N., Bockelée-Morvan, D., et al. 2013, ApJ, 774, L3

Liu, F.-C., Parise, B., Kristensen, L., et al. 2011, A\&A, 527, A19

Meier, R., Owen, T. C., Matthews, H. E., et al. 1998, Science, 279, 842

Morbidelli, A., Chambers, J., Lunine, J. I., et al. 2000, Meteor. Planet. Sci., 35, 1309

Mottram, J. C., van Dishoeck, E. F., Schmalzl, M., et al. 2013, A\&A, 558, A126

Müller, H. S. P., Thorwirth, S., Roth, D. A., \& Winnewisser, G. 2001, A\&A, 370, L49

Mumma, M. J., \& Charnley, S. B. 2011, ARA\&A, 49, 471

Ossenkopf, V., \& Henning, T. 1994, A\&A, 291, 943

Parise, B., Simon, T., Caux, E., et al. 2003, A\&A, 410, 897

Parise, B., Caux, E., Castets, A., et al. 2005, A\&A, 431, 547
Persson, M. V., Jørgensen, J. K., \& van Dishoeck, E. F. 2012, A\&A, 541, A39

Persson, M. V., Jørgensen, J. K., \& van Dishoeck, E. F. 2013, A\&A, 549, L3

Pickett, H. M., Poynter, R. L., Cohen, E. A., et al. 1998, J. Quant. Spectr. Radiat. Transf., 60, 883

Prodanović, T., Steigman, G., \& Fields, B. D. 2010, MNRAS, 406, 1108

Sandell, G., Aspin, C., Duncan, W. D., Russell, A. P. G., \& Robson, E. I. 1991, ApJ, 376, L17

Schöier, F. L., Jørgensen, J. K., van Dishoeck, E. F., \& Blake, G. A. 2002, A\&A, 390, 1001

Schöier, F. L., Jørgensen, J. K., van Dishoeck, E. F., \& Blake, G. A. 2004, A\&A, 418,185

Schöier, F. L., van der Tak, F. F. S., van Dishoeck, E. F., \& Black, J. H. 2005, A\&A, 432, 369

Shu, F. H. 1977, ApJ, 214, 488

Stark, R., Sandell, G., Beck, S. C., et al. 2004, ApJ, 608, 341

Taquet, V., López-Sepulcre, A., Ceccarelli, C., et al. 2013, ApJ, 768, L29

Villanueva, G. L., Mumma, M. J., Bonev, B. P., et al. 2009, ApJ, 690, L5

Visser, R., Jørgensen, J. K., Kristensen, L. E., van Dishoeck, E. F., \& Bergin, E. A. 2013, ApJ, 769, 19

Wilson, T. L., \& Rood, R. 1994, ARA\&A, 32, 191 